\title{
Sequential intravenous-oral amoxycillin/clavulanate (Augmentin) therapy in paediatric hospital practice
}

\author{
Urs B. Schaad, Jürg Pfenninger and Joanna Wedgwood-Krucko
}

\author{
Division of Paediatric Infectious Diseases and Intensive Care Unit, Department of \\ Paediatrics, University of Berne, Inselspital, CH-3010 Berne, Switzerland
}

\begin{abstract}
The efficacy and safety of intravenous and sequential intravenous-oral clavulanatepotentiated amoxycillin therapy was evaluated in 71 hospitalized paediatric patients, one month to 16 years of age. The infections treated included peritonsillar abscess (2 patients), purulent tracheitis (1), acute epiglottitis (24), pneumonia (31), pansinusitis (4), mastoiditis (1), cellulitis (4), lymphadenitis (2) and pyelonephritis (2). The severity of disease was rated as moderate in 31 patients $(44 \%)$, and as severe in $40(56 \%)$. Bacterial pathogens could be cultured in 26 cases $(37 \%)$. The response to therapy was prompt and followed by clinical cure in each patient. Adverse drug effects included phlebitis (in $6 \%$ ), mild gastrointestinal complaints $(6 \%)$, rash $(4 \%)$ and transient neutropenia and elevation of transaminases (one case each). It is concluded that amoxycillin/clavulanate is effective and safe treatment for bacterial infections of the respiratory tract, urinary tract, skin or soft tissues in children.
\end{abstract}

\section{Introduction}

The combination of the $\beta$-lactamase inhibitor clavulanic acid with amoxycillin provides an attractive approach to increasing the clinical utility of amoxycillin in paediatric patients, since clavulanic acid protects amoxycillin against hydrolysis by $\beta$-lactamases produced with increasing frequency by many important bacterial pathogens causing paediatric infectious diseases (Hunter et al., 1980; Reading, Farmer \& Cole, 1983). Investigators at this institution have studied the pharmacokinetics of both intravenous and oral amoxycillin/clavulanate formulations in children and have suggested dosage schedules (Schaad, Casey \& Cooper, 1983; Schaad, Casey \& Ravenscroft, 1986). Several clinical trials with oral amoxycillin/clavulanate have demonstrated that this antimicrobial combination is effective and safe therapy for various childhood bacterial infections (Jeffries, Rose \& Williams, 1983; Gooch et al., 1985; Odio et al., 1985; Jaffe et al., 1985). However, published experience with intravenous amoxycillin/clavulanate in paediatric hospital practice is scant (Price \& Horobin, 1985; Ploechl, Pirko \& Huber, 1985). Therefore, this open, non-comparative study was conducted to evaluate the efficacy and safety of intravenous and sequential intravenous-oral amoxycillin/clavulanate in the treatment of hospitalized children with various bacterial infections of moderate to severe degree.

\section{Study patients}

\section{Patients and methods}

Infants and children aged one month to 16 years admitted to the Department of Paediatrics, University of Berne, for treatment of community acquired infections of suspected 
bacterial aetiology were candidates for study. The study protocol included the following disease localizations: upper and lower respiratory tract, soft and lymphoid tissue, and urinary tract. Patients with a history of allergy to $\beta$-lactam antibiotics or underlying renal or hepatic dysfunction were excluded. The study was approved by the Institutional Committee on Human Investigations, Department of Paediatrics, University of Berne, and informed parental consent was obtained.

\section{Patient evaluation}

Complete medical history, comprehensive physical examination and suitable laboratory, radiological and microbiological studies were performed on each patient. Patients with findings suggesting non-bacterial disease were excluded from enrollment. In-vitro susceptibilities of significant pathogens to amoxycillin/clavulanate, amoxycillin and other antimicrobial agents were determined by a standardized Kirby-Bauer paper disc technique.

\section{Treatment}

Amoxycillin/clavulanate (Augmentin) vials containing $550 \mathrm{mg}$ (500 mg of amoxycillin plus $50 \mathrm{mg}$ of clavulanic acid) or $1.1 \mathrm{~g}(1 \mathrm{~g}$ of amoxycillin plus $100 \mathrm{mg}$ of clavulanic acid) were used. All patients were started on intravenous amoxycillin/clavulanate at a recommended dosage according to severity of disease of 110 to $220 \mathrm{mg}$ of amoxycillin/clavulanate/ $\mathrm{kg}$ body weight/day, divided into four equal doses administered as bolus iv injection over $2-5 \mathrm{~min}$. Once an unequivocal clinical response had been established and provided that gastrointestinal function was normal, the patients were changed to oral amoxycillin/clavulanate therapy. Paediatric oral amoxycillin/clavulanate formulations (syrup sachets or bottled suspensions) were used and contained four parts of amoxycillin and one part of clavulanic acid. Recommended dosages were between 50 and $100 \mathrm{mg}$ of amoxycillin/clavulanate $/ \mathrm{kg}$ body weight/day, divided into three equal doses. Duration of therapy was decided by the physician responsible. The remaining medical therapy was according to standard procedures.

\section{Evaluation of therapy}

Clinical, bacteriological and radiological responses to treatment were evaluated in each patient. Clinical cure was defined as complete resolution of symptoms with significant improvement of physical signs. Follow-up bacteriological cultures were done at the discretion of the physician. Follow-up chest radiographs were performed in all pneumonia patients. Adverse or toxic reactions to amoxycillin/clavulanate were assessed by daily clinical observation and haematological and biochemical monitoring at the beginning and end of treatment.

\section{Results}

\section{Patient characteristics and therapies}

From March 1985 to February 1986 a total of 71 infants and children were enrolled in the study. Their sex and age distribution and the daily dosage and duration of iv and oral amoxycillin/clavulanate therapy are listed in Table $\mathbf{I}$. 
Table I. Patient characteristics, daily dosage and duration of amoxycillin/clavulanate therapy

\begin{tabular}{|c|c|c|}
\hline Characteristic & No. patients (\%) & Mean (range) \\
\hline \multicolumn{3}{|l|}{$\operatorname{Sex}$} \\
\hline Male & $45(63)$ & \\
\hline Female & $26(37)$ & \\
\hline Age & & $411 / 12(1 / 12-159 / 12)$ \\
\hline $1-11$ months & $8(11)$ & \\
\hline $1-6$ years & $44(62)$ & \\
\hline$>6$ years & $19(27)$ & \\
\hline \multicolumn{3}{|c|}{ Daily dosage of amoxy- } \\
\hline & cillin/clavulanate & \\
\hline iv (in $4 \times /$ day) & $71(100)$ & $174(76-272)$ \\
\hline po (in $3 \times /$ day) & $64(90)$ & $60(23-110)$ \\
\hline \multicolumn{3}{|c|}{ Duration of therapy (days) } \\
\hline iv & & $4 \cdot 6(1-14)$ \\
\hline po & & $7 \cdot 2(2-15)$ \\
\hline Total & & $11 \cdot 1(5-25)$ \\
\hline
\end{tabular}

In 16 patients $(23 \%)$ the medical history revealed an underlying disease potentially relevant to the outcome of the infection. Five patients with pneumonia and two with pansinusitis had psychomotor retardation. Gastrooesophageal reflux was present in four patients with pneumonia and congenital heart disease could have promoted pneumonia in two infants. There was one case each with the association of bilateral vesicoureteric reflux and pyelonephritis, surgically repaired cleft palate and epiglottitis, and acute lymphoblastic leukaemia and pneumonia.

Nineteen patients $(27 \%)$ had received unsuccessful antimicrobial therapy either po (18 patients) or iv (one patient) prior to amoxycillin/clavulanate. In 15 cases the duration of oral antibiotic administration was only one (12 patients) or two days (three patients) and their clinical non-response was interpreted as due to inadequate duration or an unsuitable route. Three of these patients had received oral amoxycillin/clavulanate. The choice of an unsuitable antibiotic was felt to explain the clinical failure in two patients: one had received oral co-trimoxazole for cervical lymphadenitis, and one chloramphenicol for bronchopneumonia. In two patients, invitro resistance of the pathogen was the probable explanation: Haemophilus influenzae resistant to erythromycin causing lobar pneumonia in one of these, and Escherichia coli resistant to cefuroxime causing aspiration bronchopneumonia in the other.

Five patients $(7 \%)$ received other antibiotics in addition to amoxycillin/clavulanate. Three patients with pneumonia and positive cold agglutinins received oral erythromycin until negative complement fixation tests for Mycoplasma pneumoniae were reported after 3,7 and 10 days. One patient with severe, culture-negative pansinusitis and a subperiosteal abscess in the right orbital roof was concomitantly treated with clindamycin iv and the fifth patient with known bilateral vesicoureteral reflux who had developed severe pyelonephritis due to $E$. coli also received amikacin iv. This urine pathogen was sensitive in vitro to amoxycillin/clavulanate and amikacin, but resistant to amoxycillin.

In the 24 patients with acute epiglottitis immediate nasotracheal intubation was performed under deep inhalation anaesthesia as described in detail elsewhere (Gerber 
\& Pfenninger, 1986). Mean duration of intubation was $23.6 \mathrm{~h}$ with a range from 14 to $38 \mathrm{~h}$. There was no complication related to anaesthesia or intubation procedures.

\section{Infections}

The various infections requiring antibiotic treatment and their severity are listed in Table II. The degree of illness was rated as moderate in $31(44 \%)$ and as severe in 40 patients $(56 \%)$.

Surgical drainage of pus was only necessary in the case of orbital subperiosteal abscess complicating pansinusitis. Thoracocentesis was performed in all six patients with pneumonia and pleural effusion (pleuropneumonia): sterile exudates were found and in no case was there empyema. There was one case of periorbital and three cases of buccal cellulitis.

Table II. Clinical details of cases

\begin{tabular}{lrlcc}
\hline & & & \multicolumn{2}{c}{ Severity of disease } \\
Diagnosis & No. patients (\%) & Moderate & Severe \\
\hline Peritonsillar abscess & 2 & $(3)$ & 1 & 1 \\
Purulent tracheitis & 1 & $(1 \cdot 5)$ & 1 & - \\
Acute epiglottitis & 24 & $(34)$ & - & 24 \\
Pleuropneumonia & 6 & $(9)$ & 2 & 4 \\
Lobar pneumonia & 15 & $(21)$ & 10 & 5 \\
Bronchopneumonia & 10 & $(14)$ & 7 & 3 \\
Pansinusitis & 4 & $(5)$ & 3 & 1 \\
Mastoiditis & 1 & $(1 \cdot 5)$ & - & 1 \\
Cellulitis & 4 & $(5)$ & 4 & - \\
Lymphadenitis & 2 & $(3)$ & 2 & - \\
Pyelonephritis & 2 & $(3)$ & 1 & 1 \\
Total & $71(100)$ & 31 (44) & $40(56)$ \\
\hline
\end{tabular}

\section{Bacteriology}

Bacterial pathogens could be cultured from relevant samples in 26 patients (37\%). The isolated pathogens, their source and in-vitro susceptibility, as well as the infections they caused are shown in Table III. All bacterial isolates were susceptible in vitro to amoxycillin/clavulanate but four strains were $\beta$-lactamase-positive and resistant to amoxycillin: one Staphylococcus epidermidis strain, two E. coli strains, and one Klebsiella pneumoniae strain.

\section{Response to therapy}

Response to amoxycillin/clavulanate therapy was prompt in each patient and clinical assessment at the end of treatment and at follow-up revealed cure in all cases. Followup radiographs in the patients with pneumonia, sinusitis and mastoiditis showed marked resolution of the pathologic radiographic signs before amoxycillin/clavulanate therapy was discontinued. Because of clinical cure, follow-up cultures were only performed in two urine (pyelonephritis) and two pus samples (mastoiditis, cellulitis): both urine and both clear aspirate samples were culture-negative. 


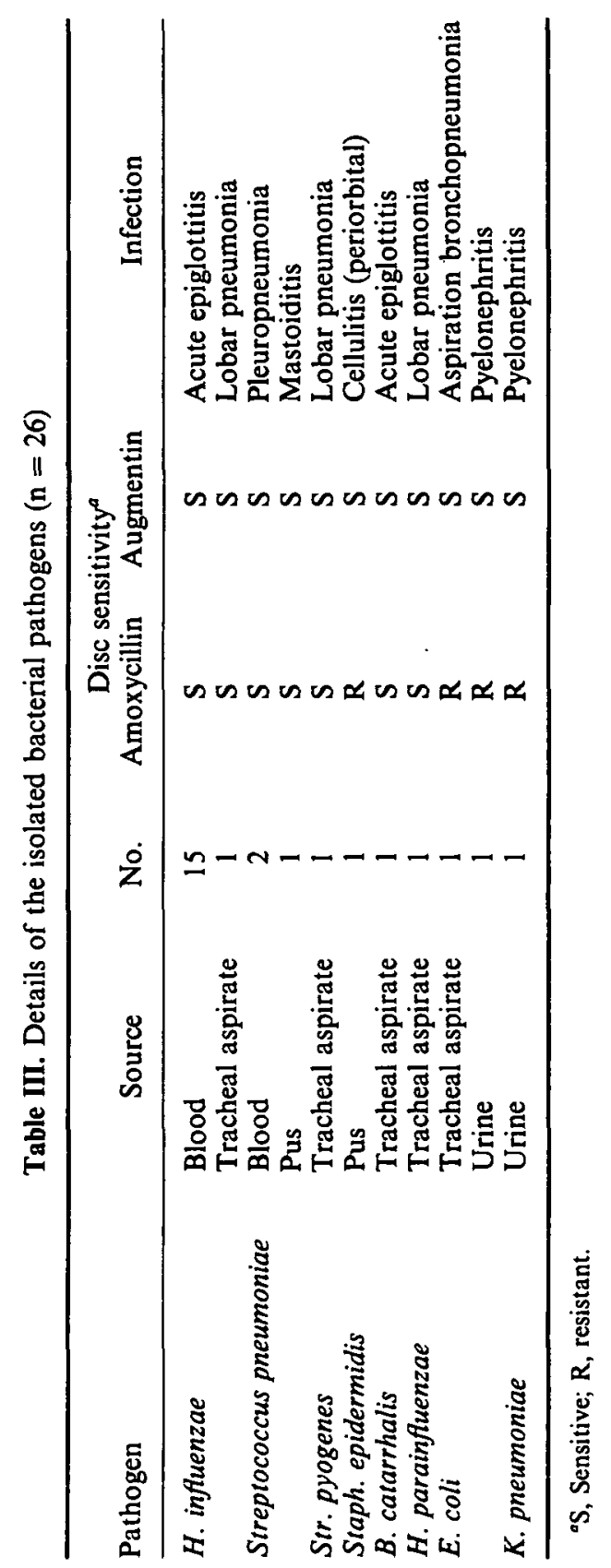


Safety

Clinical adverse effects attributed to amoxycillin/clavulanate were observed in 12 patients $(17 \%)$. Phlebitis at the injection site in four patients required change of intravenous access. Four patients experienced gastrointestinal complaints (two with diarrhoea and vomiting, two with diarrhoea only). However, these were mild side effects, and in no case was medication discontinued. Three patients developed maculopapular rashes: at the end of therapy in two cases (days 8 and 14, respectively), and in one case manifest from day 4 to day 10 of treatment. One patient developed a generalized urticarial rash on the eighth day of treatment requiring cessation of therapy.

Complete laboratory monitoring at the beginning and at the end of treatment was performed in 38 patients ( $54 \%$ ). Neutropenia developed in one patient with periorbital cellulitis (248 neutrophils/ $\mu$ l on day 6 of therapy) and resolved within three weeks. A four- and ten-fold rise respectively of the liver enzymes SGOT and SGPT was found in one patient with lobar pneumonia associated with recurrence of leukaemia and resolved partially after one week and completely after four weeks.

\section{Discussion}

Over the last ten years increasing numbers of $\beta$-lactamase-producing bacterial pathogens ( $H$. influenzae, $H$. parainfluenzae, Branhamella catarrhalis, staphylococci and Enterobacteriaceae) have been isolated from paediatric patients with various infections. Management of these conditions requires an antimicrobial agent that is resistant to cleavage by the $\beta$-lactamases and effective against both Gram-positive and Gram-negative bacteria. In addition to satisfying these basic in-vitro requirements, amoxycillin/clavulanate has other major advantages: the amoxycillin component has a long record of extensive clinical use with favourable results; the two drug constituents amoxycillin and clavulanic acid are pharmacokinetically compatible after iv and oral administration and adequate dosage schedules have been established; finally, both intravenous and oral therapy are possible with the same antibacterial agents (Schaad et al., 1983; Schaad et al., 1986).

In the present non-comparative study the efficacy and safety of intravenous and sequential intravenous-oral amoxycillin/clavulanate therapy for various childhood bacterial infections were evaluated in 71 patients. An underlying disease was present in $23 \%$, unsuccessful antimicrobial therapy prior to amoxycillin/clavulanate had been administered in $27 \%$, and the degree of illness at hospital admission was rated as severe in $56 \%$ and as moderate in $44 \%$. The response to amoxycillin/clavulanate therapy was prompt in each patient and all were cured of their infections.

These excellent clinical and bacteriological results with amoxycillin/clavulanate in paediatric hospital practice compare favourably with previous reports with oral amoxycillin/clavulanate in general paediatric practice (Jeffries et al., 1983), in childhood pneumonia (Gooch et al., 1985), otitis media (Odio et al., 1985) and skin and soft tissue infections (Jaffe et sl., 1985). Also the limited data reported with intravenous amoxycillin/clavulanate in paediatric infections (Price \& Horobin, 1985; Ploechl et al., 1985) are in agreement with the findings in this study.

Clinical adverse effects attributed to amoxycillin/clavulanate were infrequent and usually mild: in only one case of urticarial rash was cessation of therapy necessary. The observed $6 \%$ frequency of gastrointestinal complaints (diarrhoea with and without 
vomiting) with amoxycillin/clavulanate lies at the lower end of frequencies reported elsewhere: $2 \cdot 5 \%$ (Price \& Horobin, 1985), 8\% (Jeffries et al., 1983), 9\% (Jaffe et al., 1985), 15\% (Gooch et al., 1985), and 24\% (Odio et al., 1985). A rash is observed in 1 to $5 \%$ of children who receive aminopenicillins (McCracken, 1983); in this study four patients $(6 \%)$ developed rashes most probably related to drug therapy. There was one case each of transient neutropenia and temporary increase of hepatic enzymes. Whether the neutropenia and disturbed hepatic function were due solely to amoxycillin/clavulanate or to a multiplicity of factors is uncertain.

It is concluded that intravenous and sequential intravenous-oral amoxycillin/clavulanate are safe and highly effective treatments for paediatric patients admitted to hospital with respiratory tract, urinary tract, skin and soft tissue infections.

\section{Acknowledgements}

This study was supported by a grant from Beecham AG Berne, Switzerland. The authors gratefully acknowledge the cooperation of Dr P. A. Casey and $\operatorname{Dr} W$. Buechi, Beecham AG, Berne, Switzerland.

\section{References}

Gerber, A. C. \& Pfenninger, J. (1986). Acute epiglottitis: management by short duration of intubation and hospitalisation. Intensive Care Medicine 12, (in press).

Gooch, W. M., Congeni, B. L., Swenson, E. \& Snellman, L. W. (1985). Effectiveness and safety of Augmentin in the management of pneumonia of infants and children. Current Therapeutic Research 37, 178-83.

Hunter, P. A., Coleman, K., Fisher, J. \& Taylor, D. (1980). In vitro synergistic properties of clavulanic acid, with ampicillin, amoxycillin and ticarcillin. Journal of Antimicrobial Chemotherapy 6, 455-70.

Jaffe, A. C., O'Brien, C. A., Reed, M. D. \& Blumer, J. L. (1985). Randomized comparative evaluation of Augmentin and cefaclor in pediatric skin and soft-tissue infections. Current Therapeutic Research 38, 160-8.

Jeffries, M. G., Rose, A. J. \& Williams, H. N. (1983). An initial assessment of Augmentin for the treatment of paediatric infections in general practice. British Journal of Clinical Practice 37, 61-6.

McCracken, Jr., G. H. (1983). Comparative evaluation of the aminopenicillins for oral use. Pediatric Infectious Disease 2, 317-20.

Odio, C. M., Kusmiesz, H., Shelton, S. \& Nelson, J. D. (1985). Comparative treatment trial of Augmentin versus cefaclor for acute otitis media with effusion. Pediatrics 75, 819-26.

Ploechl, E., Pirko, P. \& Huber, E. G. (1985). Clinical experience with injectable Augmentin in the treatment of pediatric infections. Proceedings 4th Mediterranean Congress on Chemotherapy, Rhodes, 1984. Chemioterapia 4, Suppl 2, 853-4.

Price, J. D. \& Horobin, J. C. (1985). Intravenous Augmentin in the treatment of serious infections. Proceedings 4th Mediterranean Congress on Chemotherapy, Rhodes, 1984. Chemioterapia 4, Suppl. 2, 218-20.

Reading, C., Farmer, T. \& Cole, M. (1983). The $\beta$-lactamase stability of amoxycillin with the $\beta$-lactamase inhibitor, clavulanic acid. Journal of Animicrobial Chemotherapy 11, 27-32.

Schaad, U. B., Casey, P. A. \& Cooper, D. L. (1983). Single-dose pharmacokinetics of intravenous clavulanic acid with amoxicillin in pediatric patients. Antimicrobial Agents and Chemotherapy 23, 252-5.

Schaad, U. B., Casey, P. A. \& Ravenscroft, A. T. (1986). Pharmacokinetics of a syrup formulation of amoxycillin-potassium clavulanate in children. Journal of Antimicrobial Chemotherapy 17, 341-5.

(Manuscript accepted 2 October 1986) 